Revista Paulista de Enfermagem

Como citar este artigo

Gomes HLM, Monteiro IOP, Pina RMP, Toledo NN

de Almeida GS.

[Enfrentamento,

Dificuldades e Práticas de Autocuidado de Pacientes com Doença Renal

Crônica Submetidos à Diálise Peritoneal]. Rev Paul Enferm [Internet]. 2019;30:
Autor Correspondente

Iago Orleans Pinheiro Monteiro

E-mail:

orleansiago@hotmail.com

Endereço:

Rua Boa Vida, 223

Coroado. Manaus/AM

Fone: (92) 99154-3443.

\section{Enfrentamento, Dificuldades e Práticas de Autocuidado de Pacientes com Doença Renal Crônica Submetidos à Diálise Peritoneal}

\author{
Enfrentamiento, Dificultades y Prácticas de Autocuidado de Pacientes con \\ Enfermedad Renal Crónica en Diálisis Peritoneal \\ Coping, Difficulties and Self-care practices of Patients with Chronic Renal \\ Failure Submitted to Peritoneal Dialysis
}

\author{
Hanna Lorena Moraes Gomes ${ }^{1}$, Iago Orleans Pinheiro Monteiro², \\ Rizioléia Marina Pinheiro Pina ${ }^{3}$, Noeli das Neves Toledo ${ }^{4}$, \\ Gilsirene Scantelbury de Almeida ${ }^{5}$
}

\section{Enfermeira. Mestranda em Enfermagem. Escola de Enfermagem de Manaus/ Universidade Federal do Amazonas. \\ Graduando em Enfermagem. Escola de Enfermagem de Manaus/ Universidade Federal do Amazonas. \\ Doutora. Professora da Escola de Enfermagem de Manaus/ Universidade Federal do Amazonas. Doutora. Professora da Escola de Enfermagem de Manaus/ Universidade Federal do Amazonas. Doutora. Professora Adjunta da Escola de Enfermagem de Manaus/ Universidade Federal do Amazonas.}

\section{RESUMO}

Objetivos: Compreender o enfrentamento, as dificuldades e as práticas de autocuidado utilizadas por pessoas com doença renal crônica, submetidas ao tratamento por diálise peritoneal, por meio de uma síntese de estudos, primários e elegíveis, encontrados na literatura. Resultados: Foram selecionados 09 artigos provenientes das bases de dados LILACS, IBECS e BDENF, com base nos critérios de inclusão e exclusão. Sendo, quatro na língua portuguesa, dois na língua inglesa e três na língua espanhola. Os resultados foram discutidos em categorias específicas. Conclusão: A experimentação de um novo momento da vida do individuo acometido por Doença Renal Crônica que consequentemente, o leva ao tratamento por Diálise Peritoneal. Essa doença traz grandes mudanças no estilo de vida do acometido. Toda essa situação deve ser muito bem considerada pelo profissional de enfermagem que será o principal intermediador para o êxito nesse processo de tratamento. É esse profissional de saúde que deverá dá suporte para a família do enfermo e conduzir o paciente a receber um tratamento com todos os cuidados necessários para obter um bem-estar físico e emocional em sua nova etapa de vida.

Descritrores: Enfermagem, Insuficiência Renal Crônica, Diálise Peritoneal, Autocuidado 


\section{ABSTRACT}

Objective: To understand the coping, difficulties and self-care practices used by persons with chronic renal failure, submitted to peritoneal dialysis treatment, by a synthesis of primary and eligible studies, found in the literature. Results: Selected nine articles from the databases based on the inclusion and exclusion criteria. There are four in the Portuguese language, two in the English language and three in the Spanish language. The results were discussed in specific categories. Conclusion: The experience of a new moment of the individual's life affected by Chronic Renal Disease, which consequently leads him to Peritoneal Dialysis Treatment. This disease brings major changes in the affected individual's life style. The nursing professional who will be the main intermediator for the success in this treatment process should very well consider this whole situation. This health professional is who should give support for the family of the ill person and lead the patient to receive a treatment with all the care needed to reach a physical and emotional well-being in its new stage of life. Descriptors: Nursing, Chronic Renal Failure, Peritoneal Dialysis, Self-care.

\section{RESUMEN}

Objetivos: Comprender el enfrentamiento, las dificultades, y las prácticas de autocuidado utilizadas por personas con enfermedad renal crónica en diálisis peritoneal, por medio de una síntesis de estudios primarios y elegibles, encontrados en la literatura. Resultados: Fueron seleccionados 09 artículos provenientes de las bases de datos LLACS, IBECS y BDENF, con base en los criterios de inclusión y exclusión. Fueron cuatro artículos en portugués, dos en inglés y tres en español. Los resultados fueron discutidos en categorías específicas. Conclusión: La experiencia de un nuevo momento de la vida del individuo con Enfermedad Crónica Renal que como consecuencia lo lleva al tratamiento con Diálisis Peritoneal. Esta enfermedad trae grandes cambios en el estilo de vida de la persona que la padece. Toda esta situación debe ser ampliamente considerada por el profesional de enfermería que será el principal intermediario para el éxito de este proceso de tratamiento. Es el profesional de salud quien deberá dar soporte a la familia de la persona enferma y conducir al paciente a recibir un tratamiento con todos los cuidados necesarios para obtener un bienestar físico y emocional en esta nueva etapa de su vida.

Descriptores: Enfermería, Insuficiencia Renal Crónica, Diálisis Peritoneal, Autocuidado.

\section{INTRODUÇÃO}

A Doença Renal Crônica (DRC) é definida como anormalidades da estrutura e/ou função dos rins com implicação para a saúde, presentes por mais de três meses. A avaliação da taxa de filtração glomerular e a albuminúria são parâmetros valiosos para determinar as condutas terapêuticas na DRC e deve ser realizada anualmente com uma frequência de medição individualizada na história do paciente e em condições específicas a avaliação freqüente. ${ }^{(1)}$

A menor TFG e maior albuminúria estão associadas a um aumento na taxa de progressão e são sinérgicos. Outrossim, medidas mais recorrentes de eliminação glomerular frequente (eGFR) e albuminúria devem ser consideradas em pacientes com TFG menor e albuminúria maior. Uma vez que estas pessoas têm maior probabilidade de progredir para o agravamento da DRC. ${ }^{(1)}$

No primeiro estágio da perda da função renal há uma redução funcional de $25 \%$. No segundo, ocorre a insuficiência renal, com perda de 75\% desta capacidade. Já no terceiro, se instala a falência renal, quando a função fica abaixo de $20 \%$ e há a presença de ureia ou de outros compostos nitrogenados no sangue (azotemia) intensa. E enfim, no quarto estágio, também chamado de terminal, aparece a síndrome urêmica, sendo então necessária a terapia de diálise ou o transplante renal (TR). ${ }^{(1-2)}$

Diante da instalação do processo na DRC, a diálise peritoneal é uma das possibilidades de Terapia de Substituição renal, na qual consiste em instalar um cateter cirurgicamente no 
peritônio do usuário. Esse cateter é manipulado para injetar o líquido de diálise que precisa manter-se de 4 até 6 horas no abdome, mas propriamente no peritônio e depois é retirado. ${ }^{(3-4)}$

Atualmente existem vários centros de tratamento por todo o país. A diálise peritoneal (DP) então é indicada como a melhor opção de tratamento pelos benefícios trazidos pela manutenção ou melhoria da qualidade de vida do cliente e pelos custos mais baixos. A sua utilização ainda é menor se comparada com outras modalidades de tratamento, como a hemodiálise, por exemplo. ${ }^{(5)}$ Essa realidade pode estar associada à escassez de profissionais especializados e à carência de políticas de saúde, além de incentivo para esse tipo de tratamento. ${ }^{(6-7)}$

Apesar dos benefícios comparados aos outros tratamentos dialíticos, não se pode negar os impactos causados na vida do indivíduo submetido à DP. Ela envolve complicações em sua vida no dia a dia e exige uma participação ativa do mesmo, colocando esse paciente como sujeito vital para o seu autocuidado. ${ }^{(7)}$

Dessa forma, salienta-se que o autocuidado pode ser definido como o poder ou a competência ou até mesmo, o potencial da pessoa para esforçar-se no próprio cuidado, permitindo ao paciente ter domínio sobre seu tratamento e ter a consciência do seu próprio cuidado. ${ }^{(8-9)}$

Na DP, as práticas de autocuidado, desenvolvidas pelo indivíduo, se referem ao cuidado com o cateter e seu entorno, à terapia medicamentosa correta, à adesão à dieta e à limitação dos líquidos, mantendo vigilância necessária e prevenindo assim, possíveis complicações. Nesse contexto, os enfermeiros têm importante atuação, tendo em vista, serem profissionais que executam práticas de cuidado acolhedoras e integrais. Eles ponderam sobre os determinantes sociais, frente à assistência aos doentes crônicos. ${ }^{(10)}$

Ademais, executam importante papel educativo, pois possuem o perfil mais adequado para capacitar e preparar os clientes e familiares para atuarem de forma eficaz, diante dos procedimentos de diálise, como também, responsabilizar cada indivíduo pelo seu cuidado, seja com ajuda da família ou de cuidadores. Portanto, a atuação do profissional deve objetivar o fortalecimento de estratégias educativas que informem o usuário e seus pares sobre sua doença, sinais, sintomas, hábitos de vida saudável e cuidados com a terapêutica, conforme suas condições e necessidades, considerando o déficit de conhecimento que apresentem no decorrer do tratamento no que concerne a adaptação à diálise peritoneal. (11-12)

Considerando a realidade destacada, propôs-se um estudo que compreendesse os fatores inerentes ao indivíduo em DP, com a intenção de gerar dados que subsidiem a melhor prática de enfermagem frente ao tratamento, com destaque às práticas educativas. Assim, partiu-se da questão norteadora: "Qual o enfrentamento, dificuldades e práticas de autocuidado utilizadas por pessoas com Doença Renal Crônica em tratamento por diálise peritoneal?". Desse modo, objetivou-se compreender o enfrentamento, as dificuldades e as práticas de autocuidado, utilizadas por pessoas com DRC que são submetidas ao tratamento por diálise peritoneal, através de uma síntese de estudos primários e elegíveis encontrados na literatura.

\section{MÉTODOS}

Estudo do tipo revisão integrativa, fundamentado em cinco etapas: Formulação do problema; Coleta de dados ou definições para busca na literatura; Avaliação dos dados; Análise dos dados e; Apresentação e interpretação dos resultados. A sequência destas etapas seguiu o rigor científico exigido para os estudos em enfermagem e possibilitou a construção de conclusões por meio da síntese de vários estudos para a compreensão do tema analisado. ${ }^{(13-14)}$

A busca nas bases de dados se deu através da estratégia PICo*. Dessa forma, a questão de investigação da presente revisão foi elaborada de acordo com os tópicos propostos na estratégia. (Quadro 1) 
Quadro 1 - Estratégia de busca PICo

\begin{tabular}{|c|c|c|}
\hline & PICo & Descritores \\
\hline P (Público-alvo) & Pessoas com Doença Renal Crônica & Insuficiência Renal Crônica \\
\hline I (Interesse) & $\begin{array}{r}\text { Enfrentamento, dificuldades e as práticas } \\
\text { de autocuidado }\end{array}$ & $\begin{array}{c}\text { Autocuidado } \\
\text { Enfermagem }\end{array}$ \\
\hline Co (Contexto) & Tratamento por diálise peritoneal & Diálise Peritoneal \\
\hline
\end{tabular}

A busca dos estudos foi realizada por meio de descritores controlados por Descritores em Ciências da Saúde (DeCS)**: Enfermagem; Autocuidado; Diálise Peritoneal; Insuficiência Renal Crônica. As bases de dados utilizadas para a pesquisa foram: Literatura Latino-Americana e do Caribe em Ciências da Saúde (LILACS); Índice Bibliográfico Espanhol de Ciências da Saúde (IBECS); Base de Dados em Enfermagem (BDENF). A escolha das bases de dados foi devido aos estudos primários terem sido encontrados apenas nas mesmas. Já a combinação dos descritores foi através do operador booleano AND e se realizou de diferentes maneiras, com o intuito de ampliar as busca pelos estudos. (Quadro 2)

Quadro 2 - Combinação dos descritores que possibilitou a identificação dos estudos primários na revisão integrativa - Manaus, AM, Brasil, 2017.

\begin{tabular}{|c|c|}
\hline Base de Dados & Descritores \\
\hline Lilacs & "Enfermagem", "Autocuidado" e "Diálise Peritoneal" \\
\hline IBECS & "Enfermagem", "Insuficiência Renal Crônica", "Autocuidado" e "Diálise Peritoneal" \\
\hline BDENF & "Insuficiência Renal Crônica", "Autocuidado" e "Diálise Peritoneal" \\
\hline
\end{tabular}

Estudos publicados nos últimos 15 anos, que descreviam o enfrentamento, as dificuldades e o autocuidado de pacientes com Doença Renal Crônica, sobre pacientes submetidos ao tratamento por diálise peritoneal e estudos elegíveis publicados nos idiomas Português, Inglês e Espanhol foram os critérios de inclusão para esta revisão integrativa. Foram excluídos os estudos que se enquadravam nos seguintes critérios: dissertações, teses, monografias e estudos primários de periódicos sem acesso livre. Foram realizadas leituras dos títulos, bem como dos resumos de todos os estudos primários elegíveis e conforme o critério de seleção da revisão integrativa estudos foram selecionados e lidos na íntegra.

Os estudos foram classificados de acordo com as abordagens metodológicas quantitativas e qualitativas. A verificação dos resultados salientados foi realizada de modo descritivo, apresentando assim, uma síntese de cada estudo incluído na revisão integrativa. Para a discussão dos resultados, levantaram-se categorias específicas que atendessem ao objetivo do estudo e considerassem o abordado nos artigos. Portanto, as categorias foram: Enfrentamento à DRC e ao tratamento por DP; Dificuldades enfrentadas por doentes renais crônicos em tratamento por DP; Práticas de autocuidado por pacientes com DRC em tratamento por DP.

\section{RESULTADOS E DISCUSSÃO}

A busca dos estudos primários foi realizada no mês de novembro de 2017 e os resultados são descritos da Figura 1. 


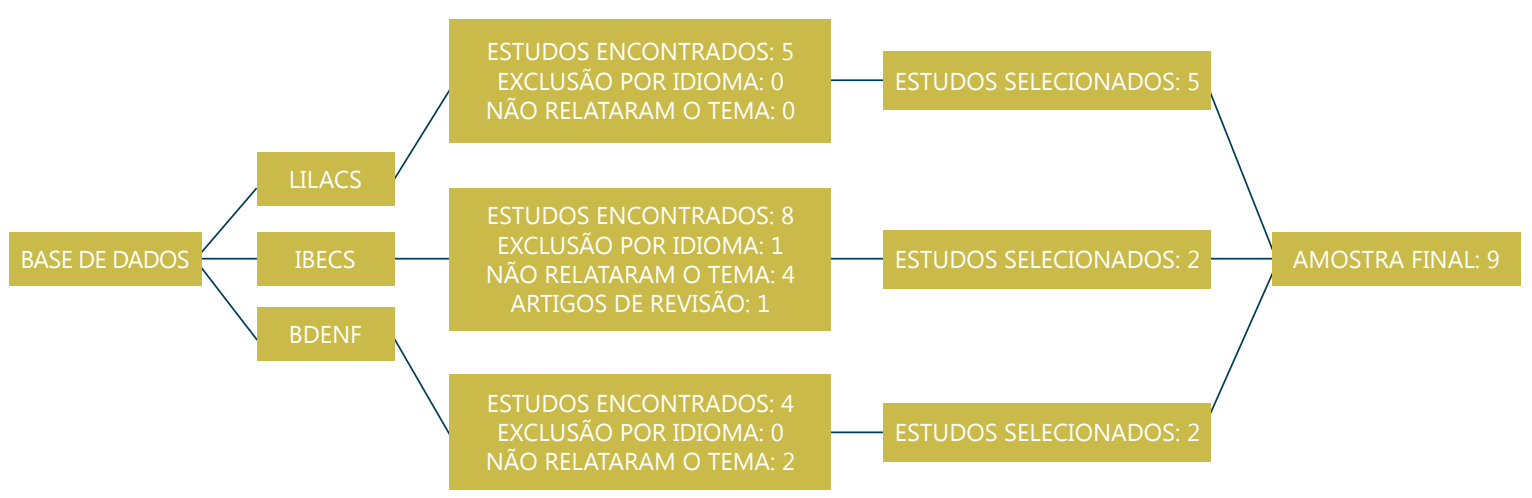

Figura 1 - Fluxograma da seleção dos estudos primários elegíveis e motivos de exclusão

Dos 09 estudos primários incluídos na revisão, quatro foram publicados no idioma português, dois nos idiomas inglês e espanhol, respectivamente. Houve diversidade em relação aos periódicos, sendo que cada estudo primário incluído foi publicado em um periódico diferente, quanto aos tipos de estudos, houve predominância de estudos qualitativos, com apenas um estudo quantitativo. O Quadro 3 mostra a representação geral dos estudos.

Quadro 3 - Representação dos estudos primários incluídos na revisão integrativa de literatura

\begin{tabular}{|c|c|c|c|c|}
\hline $\begin{array}{c}\text { Bases de } \\
\text { dados }\end{array}$ & Título do artigo & Autores & Periódico & Objetivos \\
\hline 1 - LILACS & $\begin{array}{l}\text { A dialética da } \\
\text { vida cotidiana } \\
\text { de doentes com } \\
\text { insuficiência renal } \\
\text { crônica: entre } \\
\text { o inevitável e o } \\
\text { casual. }\end{array}$ & $\begin{array}{l}\text { Machado } \\
\text { LRC, Car MR. }\end{array}$ & $\begin{array}{l}\text { Rev. esc. enferm. } \\
\text { USP vol.37 no. } \\
3 \text { São } \\
\text { Paulo Sept. } 2003\end{array}$ & $\begin{array}{l}\text { Procurou-se compreender } \\
\text { o cotidiano da vida de } \\
\text { doentes com insuficiência } \\
\text { renal crônica. }\end{array}$ \\
\hline 2 - IBECS & $\begin{array}{l}\text { Análisis de la } \\
\text { capacidade de } \\
\text { autocuidados } \\
\text { em pacientes } \\
\text { em diálises } \\
\text { peritoneal. }\end{array}$ & Algarra AJC. & $\begin{array}{l}\text { Enferm Nefrol } \\
\text { vol.18 no.1 } \\
\text { Madrid Jan./ } \\
\text { Mar. } 2015\end{array}$ & $\begin{array}{l}\text { Medir a operatividade } \\
\text { da capacidade de } \\
\text { autocuidado. }\end{array}$ \\
\hline 3 - LILACS & $\begin{array}{l}\text { Atividade da } \\
\text { vida diária dos } \\
\text { pacientes em } \\
\text { tratamento de } \\
\text { diálise peritoneal } \\
\text { intermitente com } \\
\text { cicladora }\end{array}$ & $\begin{array}{l}\text { Scatolin BE, } \\
\text { Vechi AP, } \\
\text { Ribeiro DF } \\
\text { et al. }\end{array}$ & $\begin{array}{l}\text { Arq Ciênc Saúde } \\
2010 \text { jan-mar; } \\
\text { 17(1):15-21 }\end{array}$ & $\begin{array}{l}\text { Os objetivos deste estudo } \\
\text { foram caracterizar os } \\
\text { pacientes com DRC em } \\
\text { tratamento de DPI com } \\
\text { cicladora e descrever as } \\
\text { condições de vida destes } \\
\text { pacientes. }\end{array}$ \\
\hline 4 - IBECS & $\begin{array}{l}\text { Capacidad de } \\
\text { autocuidado de } \\
\text { los pacientes em } \\
\text { diálisis peritoneal: } \\
\text { um estudio piloto } \\
\text { en Bogotá. }\end{array}$ & $\begin{array}{l}\text { Algarra AJC, } \\
\text { Díaz AJ, } \\
\text { Janeth F. }\end{array}$ & $\begin{array}{l}\text { Enferm. } \\
\text { glob. vol. } 12 \text { no. } \\
30 \text { Murcia abr. } 201\end{array}$ & $\begin{array}{l}\text { Medir a capacidade de } \\
\text { autocuidado e sua relação } \\
\text { com características } \\
\text { sociodemográficas } \\
\text { e clínicas em um } \\
\text { grupo de pacientes no } \\
\text { programa de diálise } \\
\text { peritoneal de acordo } \\
\text { com sua capacidade de } \\
\text { autocuidado. }\end{array}$ \\
\hline
\end{tabular}


Continuação do Quadro 3

\begin{tabular}{|c|c|c|c|c|}
\hline $\begin{array}{c}\text { Bases de } \\
\text { dados }\end{array}$ & Título do artigo & Autores & Periódico & Objetivos \\
\hline 5 - LILACS & $\begin{array}{l}\text { Hemodialysis: } \\
\text { patient's } \\
\text { adaptation and } \\
\text { life style. }\end{array}$ & $\begin{array}{l}\text { Barbosa GS, } \\
\text { Valadares GV. }\end{array}$ & $\begin{array}{l}\text { Acta paul. } \\
\text { enferm. vol.22 no. } \\
\text { spe1 São } \\
\text { Paulo } 2009\end{array}$ & $\begin{array}{l}\text { Identificar como o paciente } \\
\text { dependente de hemodiálise } \\
\text { enfrenta no dia a dia o } \\
\text { processo saúde-doença, } \\
\text { considerando atitudes, } \\
\text { comportamentos e práticas. }\end{array}$ \\
\hline 6 - BDENF & $\begin{array}{l}\text { Patients' } \\
\text { experiences } \\
\text { of peritoneal } \\
\text { dialysis at home: a } \\
\text { phenomenological } \\
\text { approach }\end{array}$ & $\begin{array}{l}\text { Sadala MLA, } \\
\text { Bruzos GAS, } \\
\text { Pereira ER } \\
\text { et al. }\end{array}$ & $\begin{array}{l}\text { Rev. latinoam. } \\
\text { enferm. [internet]. } \\
\text { 2012; 20(1):68-75. }\end{array}$ & $\begin{array}{l}\text { O objetivo deste estudo } \\
\text { foi compreender a } \\
\text { experiência da diálise } \\
\text { peritoneal domiciliar, a } \\
\text { partir da narrativa dos } \\
\text { pacientes }\end{array}$ \\
\hline 7 - LILACS & $\begin{array}{l}\text { Peritoneal } \\
\text { dialysis: family } \\
\text { care for chronic } \\
\text { kidnay disease } \\
\text { patients in home- } \\
\text { based treatment }\end{array}$ & $\begin{array}{l}\text { TavaresIMAB, } \\
\text { Lisboall MTL, } \\
\text { Ferreirall MA } \\
\text { et al. }\end{array}$ & $\begin{array}{l}\text { Rev Bras Enferm } \\
\text { [Internet]. } \\
2016 \text { nov- } \\
\text { dec;69(6):1107-13. }\end{array}$ & $\begin{array}{l}\text { Propor um modelo de } \\
\text { cuidado familiar ao } \\
\text { cliente renal crônico em } \\
\text { tratamento com a Diálise } \\
\text { Peritoneal a partir das } \\
\text { evidências apontadas } \\
\text { pelos familiares. }\end{array}$ \\
\hline 8 - BDENF & $\begin{array}{l}\text { Práticas de } \\
\text { autocuidado de } \\
\text { pessoas com } \\
\text { insuficiência } \\
\text { renal crônica } \\
\text { submetidas à } \\
\text { diálise peritoneal } \\
\text { ambulatorial } \\
\text { contínua }\end{array}$ & $\begin{array}{l}\text { Calderan C, } \\
\text { Torres AAP, } \\
\text { Zillmer JGV } \\
\text { et al. }\end{array}$ & $\begin{array}{l}\text { R. pesq.: cuid. } \\
\text { fundam. online } \\
\text { 2013. jan./mar. } \\
\text { 5(1):3394-02 }\end{array}$ & $\begin{array}{l}\text { Conhecer as práticas de } \\
\text { autocuidado utilizadas } \\
\text { por pessoas com } \\
\text { insuficiência renal crônica } \\
\text { submetidos à diálise } \\
\text { peritoneal ambulatorial } \\
\text { contínua (CAPD). }\end{array}$ \\
\hline 9 - LILACS & $\begin{array}{l}\text { Tratamento com } \\
\text { diálise peritoneal: } \\
\text { a prática do } \\
\text { autocuidado com } \\
\text { contexto familiar }\end{array}$ & $\begin{array}{l}\text { TavaresI } \\
\text { JMAB, } \\
\text { LisboaIIMtL. }\end{array}$ & $\begin{array}{l}\text { Ver enferm UERJ, } \\
\text { Rio de Janeiro, } \\
2015 \text { mai/jun; } \\
\text { 23(3):344-9 }\end{array}$ & $\begin{array}{l}\text { Os objetivos do estudo } \\
\text { foram identificar as } \\
\text { dificuldades enfrentadas } \\
\text { pelos clientes ao realizar } \\
\text { a diálise peritoneal } \\
\text { ambulatorial contínua } \\
\text { (DPAC) no domicílio e } \\
\text { analisar as soluções por } \\
\text { eles adotadas para lidar } \\
\text { com elas. }\end{array}$ \\
\hline
\end{tabular}

Fonte: Lilacs. Ibecs. Bdenf.

Os achados forneceram dados relevantes acerca dos aspectos que envolvem o enfrentamento, as dificuldades e as práticas de autocuidado de pacientes com DRC submetidos à Diálise Peritoneal. A discussão segue com a reflexão crítica dos achados com base no referencial teórico adotado através das categorias específicas levantadas.

\section{ENFRENTAMENTO À DRC E AO TRATAMENTO POR DP}

Discorrer sobre a maneira como os clientes enfrentam o quadro da DRC e o tratamento e como os profissionais conseguem ter uma prática de cuidado mais específica e humanizada é crucial. Sabe-se que o processo de enfrentamento de um quadro de doença crônica irá depender de diversos fatores, tais como: idade, gênero, doença, crenças, habilidades, entre outros. Para auxiliar o paciente nesse processo, o enfermeiro deve buscar compreender esses fatores, reforçando-os com vista a um quadro positivo.(15) 
Nessa conjuntura, os estudos mostram que os pacientes não questionam a sua condição, muito menos percebem o motivo do adoecimento. Para muitos pacientes, não existe o raciocínio de que atitudes outrora realizadas foram os fatores condicionantes que os levaram a desenvolver a doença. Ademais, a doença juntamente com o tratamento resulta em outro efeito negativo, a sensação de perda da identidade do próprio corpo pelas mudanças que alteram a forma como ele sempre foi visto. Portanto, a forma como a doença progride causa incerteza e medo.

Com relação ao tratamento, no estudo um, os clientes consideram o tratamento que recebem como um "presente" gratuito do governo e por isso não lhes é permitido o direito de questionar. Afinal, o governo já "faz muito" em bancar o tratamento.

Somando a isso, no estudo cinco, os pacientes passaram a enxergar o tratamento como sendo uma tortura e perda de tempo, por não conseguirem enxergar possibilidade de cura. Houve, todavia, uma mudança no quadro de aceitação, a partir do momento em que passaram a reconhecer a importância do tratamento e a enxergá-lo como a solução.

As práticas adotadas assemelhavam-se às que já se realizavam antes de iniciarem o tratamento. De modo que, alguns dias após a adaptação, os clientes já realizavam atividades sociais e de lazer, ao mesmo tempo, em que se adequavam às exigências do tratamento e à nova rotina de vida.

No estudo seis, descreve-se a inquietude frente às possibilidades de morte e a incerteza de um universo desconhecido e assustador. O tratamento proposto pela equipe seria a única maneira de sobreviver, porém, na prática, mostra-se doloroso e invasivo, limitando suas atividades sociais, além do mais, tornaram-se dependentes de um serviço indispensável até conseguir um transplante renal. Foi dessa maneira, que todos relataram suas dificuldades para se adaptar e aceitar esse novo estilo de vida. Assim, torna-se relevante que o profissional de enfermagem intervenha diante dos questionamentos acerca dos diversos fatores que envolvem o tratamento, salientando a importância que o mesmo detém para a melhora do quadro instalado.

São inúmeros os fatores que podem interferir na adesão ao recurso terapêutico do cliente submetido ao tratamento por DP, como por exemplo, o histórico de doenças crônicas do indivíduo, tendo em vista, que a aceitação do quadro pode ser influenciada pelas experiências que o doente já vivenciou, configurando parte de sua subjetividade. Ademais, compreender esse histórico, pode ser de grande relevância para elaboração de estratégias para o tratamento de doentes crônicos, sendo a principal iniciativa, o acompanhamento constante do profissional de saúde e a viabilização de atividades educativas, somadas ao contato deste com outros doentes crônicos, para que possam compartilhar suas experiências. (16) Outro aspecto importante para compreensão do enfrentamento, são estratégias educativas que levam em consideração, as vivências que o doente já carrega. ${ }^{(17)}$

Dessa forma, os resultados dos estudos mostraram uma grande incidência de história pregressa e familiar de hipertensão arterial sistêmica (HAS). A incidência da doença crônica cardiovascular é um dado que reafirma a hipertensão arterial como principal fator de risco para o surgimento de nefropatias ${ }^{(18)}$.

Em pacientes com idade acima de 65 anos, a HAS é considerada como uma das principais causas de DRC ${ }^{(2)}$. Fato que influencia no enfrentamento da doença, tendo em vista que um dos estudos constatou que os clientes nesta faixa-etária, costumam não aderir ao tratamento farmacológico e consideram seu estado de saúde como bom.

Apesar dos dados apresentados, o estudo dois mostrou que a idade não é um fator determinante para o aparecimento de doenças. Contudo, é evidente, que a função renal se deteriora ao longo dos anos ${ }^{(1)}$. Nesta perspectiva, é importante o surgimento e fortalecimento de programas destinados ao melhoramento da qualidade de vida desde uma idade mais jovem. 


\section{Dificuldades enfrentadas por doentes renais crônicos em tratamento por DP}

As pesquisas abordaram que mudanças provenientes da doença e do tratamento dialítico, vivenciadas pelos pacientes com relação às atividades desenvolvidas em seu meio social e vida diária, são causadoras de limitações. As alterações na rotina diária destes sujeitos são ainda mais evidenciadas, após início do tratamento por hemodiálise. É quando se faz necessário ir ao local de tratamento várias vezes na semana, ficando horas ligados a uma máquina. Fato esse, que causa modificações em seu comportamento, alterando sua percepção e sentimento.

Os conceitos populares concernentes ao binômio saúde-doença são diferentes do científico. Dessa forma, faz-se necessária a intervenção do profissional de enfermagem, com vista a tornar o doente ciente das alterações que irá enfrentar no quadro estabelecido. Sendo assim, é importante sua intervenção para que haja superação das dificuldades encontradas durante o tratamento. ${ }^{(19)}$

O estudo nove identificou as principais dificuldades enfrentadas pelos pacientes ao realizar a diálise peritoneal. O fato de não poder trabalhar, é um dos principais motivos de preocupação, principalmente para aqueles que eram os mantedores do sustento da família. O sentimento de limitação e a incapacidade frente à nova realidade é o que assola esses pacientes. Outra dificuldade encontrada foi o condicionamento dos materiais. Muitos viviam em domicílios pequenos e frequentemente, acompanhados de outros familiares. A sensação de tirar o conforto da família e atrapalhar o convívio familiar era outro fator preocupante.

Ainda, no que se referem às dificuldades do tratamento no domićlio, certos sujeitos da pesquisa mencionaram que possuem dificuldades com alguns membros de suas famílias, devido às limitações impostas pela condição clínica. Isso afeta toda família, pois há uma quebra do equilíbrio familiar e assim precisarão também se adaptar ao convívio com um familiar que ficou doente e precisa de $\operatorname{cuidados}^{(9-20)}$.

Neste aspecto, é importante um contexto familiar estruturado, tendo em vista o papel crucial que a família tem no processo de doença e tratamento. Sendo, na maioria dos casos, o âmbito em que o doente encontrará apoio. A família ainda se encaixa como principal aliada ao enfermeiro para execução de um plano de cuidados eficaz. ${ }^{(19)}$

Assim, existem ações que a família precisa superar em relação ao familiar doente, tais como: diminuição da ansiedade, informações de apoio por pessoal habilitado, sentimentos de solidariedade ao familiar, que só será possível se cada um vivenciar esse enfrentamento junto ao paciente (21-22). $^{2}$

\section{Práticas de autocuidado por pacientes com DRC em tratamento por DP}

A capacidade de operacionalizar o autocuidado foi evidenciada como alta por parte dos pacientes nos estudos, embora alguns participantes não aderissem ao tratamento de maneira fidedigna devido ao esquecimento. Assim, a perda de memória é um aspecto que deve ser considerado nos planos de cuidado com intervenções educativas a pacientes em DP(23).

Deve-se reconhecer também as diferenças que haverá nos indivíduos ao operacionalizar o ensino do autocuidado. Os conteúdos a serem repassados são cruciais, mas a maneira como eles serão transmitidos devem levar em conta, a subjetividade de cada um. Só assim será possível acertar na metodologia correta e obter resultados positivos ${ }^{(24)}$.

No estudo quatro, um número elevado de pessoas, reconhece a necessidade de atitudes que modifiquem suas ações de cuidados, mostrando para o doente, a relevância de reavaliarem suas atitudes com base nos processos desenvolvidos pelos profissionais da saúde (25).

A motivação que os doentes apresentam no processo de tratamento, a partir dos conhecimentos repassados pela equipe de enfermagem, perpassa também para o profissional de 
saúde, na medida em que observa a autonomia adquirida pelo paciente e, que suas habilidades como impulsionador no processo de tratamento, tem alcançado êxito na execução de práticas de autocuidado e no processo de cuidado(24).

Outro aspecto importante para o desenvolvimento das práticas de autocuidado é o apoio da família(24) . No estudo sete, objetivou-se propor um modelo de cuidado familiar ao cliente renal crônico em tratamento com a Diálise Peritoneal, a partir das evidências apontadas pelos familiares. Este foi baseado no modelo proposto por Madeline Leininger em sua Teoria da Diversidade Cultural de cuidados.

Esse cuidado fundamentado em base cultural levou ao conhecimento de como os pacientes vivem e se relacionam com seus familiares, permitindo assim que as práticas de cuidado sejam adequadas de acordo com as situações vivenciadas.

Portanto, ao utilizarem o modelo de cuidado familiar, é possível que tanto a enfermagem, quanto os familiares acessem esses conhecimentos de forma compartilhada, tornando assim mais fácil atingir um cuidado coerente com a cultura das famílias envolvidas com a diálise peritoneal.

É indispensável que o enfermeiro inclua a família no processo de cuidado, sendo grande aliada para promoção do autocuidado dos indivíduos. O enfermeiro, certamente, tem a reponsabilidade de levantar as informações sobre os conhecimentos que a família já carrega, considerando-os como agentes dotados de ferramentas que podem ser utilizadas em favor do indivíduo que necessita dos cuidados no núcleo familiar. ${ }^{(19)}$

Nos resultados apresentados pelo estudo oito, abordaram-se dois temas principais: práticas de autocuidado à doença; Práticas de autocuidado relacionadas ao tratamento por CAPD. Quanto às práticas de autocuidado à doença, estas estão relacionadas à alimentação e ingesta hídrica. Os participantes buscam balanceá-la e ingerir o que é recomendado pelos profissionais de saúde, como reduzir o consumo de sal, ingerir frutas, verduras e legumes, dentre outros. Todavia, tudo em porções fracionadas. Quanto à ingesta hídrica, teria que ser em porções limitadas e restritas nos horários das refeições e/ou durante a administração de medicamentos. Essas medidas são adotadas a fim de evitarem possíveis complicações do quadro clinico.

O objetivo de se ter uma alimentação o mais próximo do que seria adequado, está relacionado com a finalidade de proporcionar alimentos nutritivos para que ele possa satisfazer suas necessidades humanas básicas (NHB) ${ }^{(26)}$ além de serem necessárias para o sucesso do tratamento(27).

Podemos ainda destacar outra prática relacionada a satisfação de NHB, o sono. O paciente estando descansado teria maior ânimo para enfrentar as dificuldades da doença e realizar o tratamento de maneira correta. $\mathrm{O}$ ato de se ter uma qualidade de sono pode ser alterado devido a presença de situações preocupantes, como por exemplo, manutenção ao tratamento, dependência familiar e/ou de cuidadores, medo do abandono e morte. Diante disso, é possível o surgimento de sintomas depressivos ou esgotamento frente aos processos adaptativos.

Sobre práticas de autocuidado relacionadas ao tratamento por CAPD, temos: adaptações ao ambiente familiar para a realização ao tratamento, as etapas que constituem o procedimento, o cuidado com a higiene do local, lavagem das mãos, a importância em realizar o tratamento na hora marcada, o frequente uso de álcool, maneira de assegurar a assepsia do procedimento e em relação à manutenção da higiene do cateter e das práticas, como banho diário, lavagem das mãos, curativo oclusivo para proteger e o uso do álcool como forma de desinfetar os utensílios utilizados a fim de tornar seguro o procedimento.

\section{CONCLUSÃO}

Este estudo possibilitou compreender os resultados analisados e fundamentar o conhecimento acerca do enfrentamento, dificuldades e autocuidado sob a percepção dos pacientes 
frente às rotinas exigidas pela terapia dialítica. Sob esse enfoque, constatamos que tanto o enfrentamento, quanto as dificuldades estão influenciados pelas mudanças causadas pelo novo estilo de vida a ser adotado pelos indivíduos. Os resultados ainda reforçam a importância do profissional de enfermagem na orientação contínua ao paciente e à sua família para a realização e manutenção do seu autocuidado, evitando assim possíveis complicações e consequentemente melhor qualidade de vida.

Cabe lembrar a importância dos pacientes receberem orientações que lhes permitam ter uma visão crítica e reflexiva a respeito do seu estado de saúde - doença. Por isso, faz-se necessário que as orientações realizadas pela equipe de enfermagem sejam constantes e considerem os fatores subjetivos dos clientes e familiares, seu histórico, suas crenças e valores. No entanto, para que isso aconteça, é necessário que o paciente também busque sempre conhecer o seu corpo. Quanto maior for o seu conhecimento sobre o processo da doença e do tratamento, maior será o seu envolvimento no autocuidado, confirmando o alcance da sua autonomia.

Sugere-se a implementação de tecnologias educativas que poderiam abordar a temática em questão e instruir familiares, cuidadores e pacientes sobre os enfrentamentos frente à nova realidade de vida, a que esses pacientes estão submetidos. Outra proposta seria elaboração de um aplicativo para dispositivos móveis, onde familiares e/ou pacientes teriam acesso às informações sobre o seu quadro clinico, espaço especifico para tirar duvidas, opção de sala de papo com outras pessoas na mesma condição que ele, para quem sabe, dividir experiências e compartilhar sentimentos.

\section{REFERÊNCIAS}

1. Kidney Disease: Improving Global Outcomes (KDIGO) CKD Work Group. KDIGO 2012 clinical practice guideline for the evaluation and management of chronic kidney disease. Kidney Int Suppl. 2013; 3: 1-150. Disponível em: https://www.kisupplements. org/issue/S2157-1716(13)X3100-4.

2. Pecoits-Filho R, Rosa-Diez G, Gonzalez-Bedat M, Marinovich S, Fernandez S, Lugon J, et al. Tratamento substitutivo da função renal na doença renal crônica: uma atualização do Registro Latino-Americano de Diálise e Transplante. Braz. J. Nephrol. 37(1):09. Disponível em: http://bjn.org.br/details/1717/pt-BR.

3. Pedroso VSM, de Andrade GB, Weykamp JM, Cecagno D, de Medeiros AC, de Siqueira $\mathrm{HCH}$. Ações do enfermeiro na capacitação do usuário e família em diálise peritoneal. Rev. Pesqui. Cuid. Fundam. (online). 2018; 10(2): 572-576. Disponível: http://www.seer.unirio.br/index.php/cuidadofundamental/article/view/6467.

4. Nobre DDC, Soares ER, Zillmer JGV, Schwartz E, Dias ÂJS, Silva GJSD. Qualidade de vida de pessoas em diálise peritoneal. Rev. enferm. UFPE on line. 2017; 11(10): 4111-4117. Disponível em: https://periodicos.ufpe.br/revistas/revistaenfermagem/ article/viewFile/231172/25141.

5. Sesso RC, Lopes AA, Thomé FS, Lugon JR, Martins CT. Brazilian Chronic Dialysis Survey 2016. Brazilian Journal of Nephrology. 2017; 39(3): 261-266. Disponível em: http://www.scielo.br/scielo.php?script=sci_arttext\&pid=S0101-28002017000300261.

6. Rangel CHIF, Ribeiro RDCHM, Cesarino CB, Bertolin DC, Santos MCD, Mazer LE. Peritonites em pacientes com insuficiência renal crônica em tratamento de diálise peritoneal. REME rev. min. enferm. 2017; 21: 1-7. Disponível em: http://www.reme. org.br/artigo/detalhes/1196.

7. Calderan C, Zillmer JGV, Torres AAP, Schwartz E, da Silva DGV. Self-care practices for peoples with chronic renal failure undergoing continuous ambulatory peritoneal dialysis. Rev Pesqui Cuid Fundam. (Online). 2013; 5(1): 3394-3402. Disponível em: https://dialnet.unirioja.es/servlet/articulo?codigo $=5090874$. 
8. Branco JMA, Lisboa MTL. Tratamento com diálise peritoneal: a prática do autocuidado no contexto familiar. Rev enferm. UERJ. 2015; 23.3: 344-349. Disponível em: http://www.facenf.uerj.br/v23n3/v23n3a09.pdf.

9. Orem DE. Nursing: concepts of practice. 3rd ed. New York: McGraw-Hill Book Co.; 1995.

10. Becker RM, Heidemann ITSB, Meirelles BHS, Costa MFBNA, Antonini FO, Durand MK. Práticas de cuidado dos enfermeiros a pessoas com Doenças Crônicas Não Transmissíveis. Rev Bras Enferm. 2018;71(Suppl 6):2643-9, 2018. [Edição temática: Boas Práticas no Processo de Cuidado como Centralidade da Enfermagem]. Disponível em: http://www.scielo.br/scielo. php?pid=S0034-71672018001202643\&script=sci_arttext.

11. Cardoso S, Oselame GB, de Almeida Dutra D, de Oliveira EM. Diálise Peritoneal: atuação do enfermeiro aos pacientes em tratamento dialítico domiciliar. Rev Uniandrade. 2015; 16(1): 23-30. Disponível em: https://www.uniandrade.br/ revistauniandrade/index.php/revistauniandrade/article/view/175.

12. Torreão CL, de Souza SR, Aguiar BGC. Cuidados de enfermagem ao cliente em diálise peritoneal: contribuição para prática e manejo clínico. Rev. Pesqui. Cuid. Fundam. (online) 2009; 1(2). Disponível em: http://www.seer.unirio.br/index.php/ cuidadofundamental/article/view/415.

13. Crossetti MGO. Integrative review of nursing research: scientific rigor required. Rev. Gaúcha Enferm. 33(2): 12-13, 2012. Disponível em: http://www.scielo.br/scielo. php? script=sci_arttext\&pid $=$ S1983-14472012000200003.

14. Soares CB, Hoga LAK, Peduzzi M, Sangaleti C, Yonekura T, Silva D. Integrative review: concepts and methods used in nursing. Rev Esc Enferm USP. 48(2): 335-45, 2014. Disponível em: http://www.scielo.br/scielo.php?script=sci_arttext\&pid $=$ S0080-62342014000200335.

15. Paula, ES de; Nascimento, LC; Rocha, SMM. Religion and spirituality: the experience of families of children with Chronic Renal Failure. Revista brasileira de enfermagem, 62 (1):100-106, 2009. Disponível em: http://www.scielo.br/scielo. php?pid=S0034-71672009000100015\&script=sci_arttext

16. Maldaner CR, Beuter M, Brondani CM, Budó MLD, Pauletto MR. Fatores que influenciam a adesão ao tratamento na doença crônica: o doente em terapia hemodialítica. Rev Gaúcha Enferm., Porto Alegre (RS). 29(4):647-53, 2008. Diponível em: https://seer.ufrgs.br/RevistaGauchadeEnfermagem/article/view/7638

17. Toledo Melina Mafra, Rodrigues Sandra de Cássia, Chiesa Anna Maria. Educação em saúde no enfrentamento da hipertensão arterial: uma nova ótica para um velho problema. Texto contexto enferm. 16(2):233-238, 2007. Disponível em: http://www. scielo.br/scielo.php?script=sci_arttext\&pid=S010407072007000200004\&lng=en.

18. Ramirez J. Perez O, Ramirez S, Carrillo S, VargasG, Fragoso JM. Genetica y genomica de lahipertensionarterial: una actualizacion, [acesso em 2017 nov 27] disponível em: http:// zl.elsevier.es/es/revista/archivos-cardiologia-mexico-293/ genetica-genomica-hipertension-arterialuna-actualizacion-90028661-articulo-revision-2011.

19. da Silva BM, da Silva, MAA, Sezeremeta DC, Basílio G, \& Marcon, SS. Conhecimentos em saúde e dificuldades vivenciadas no cuidar: perspectiva dos familiares de pacientes em tratamento dialítico. Ciência, Cuidado e Saúde, 10(4):722-730, 2011. Disponível em: http://eduem.uem.br/ojs/index.php/CiencCuidSaude/article/ viewFile/18316/pdf

20. Freitas KS, Menezes IG, Mussi FC. Conforto na perspectiva de familiares de pessoas internadas em Unidade de Terapia Intensiva. Texto contexto enferm. 2012;21: 896-904. Disponível em: http://www.scielo.br/scielo. php?pid=S0104-07072012000400021\&script $=$ sci_abstract

21. Mota MS, Gomes GC, Petuco VM, Heck RM, Barros EJL, Gomes VLO. Facilitadores do processo de transição para o autocuidado da pessoa com estoma: subsídios para 
enfermagem. Revescenferm USP. 2015; 49:82-8. Disponível em: http://www.scielo.br/ pdf/reeusp/v49n1/pt_0080-6234-reeusp-49-01-0082.pdf

22. Bedina LF, Busanellob J, Sehnemc GD, Silva FM, Poll MA. Estratégias de promoção da autoestima, autonomia e autocuidado das pessoas com feridas crônicas. Rev Gaúcha Enferm. 35:61-7, 2014. Disponível em: https://seer.ufrgs.br/

RevistaGauchadeEnfermagem/article/view/43581

23. Portoles J. Coronel F. Dialisis peritoneal ensituacionesespeciales. [Internet] [consultado El 25 de noviembre de 2017] disponível em: http://www. valladoliddeporte.es/fasciculo/2012/06/ejercicio-para-losenfermos-de-rinon-

24. Figueiredo $A E$, Kroth, LV, Lopes MHI. Diálise peritoneal: educação do paciente baseada na teoria do autocuidado. Science Medical, 15(3): 198-202, 2005. Disponível em: http://revistaseletronicas.pucrs.br/ojs/index.php/scientiamedica/article/ viewDownloadInterstitial/1567/7946

25. Mendez A. Epidemiologia de la insuficiencia renal crônica em Mexico. Dialisis y Trasplante. 31:7-11, 2010. Disponível em: http://www.elsevier.es/ es-revista-dialisis-trasplante-275-articulo-epidemiologia-insuficiencia-renal-cronicamexico-S1886284510700047

26. Landim CAP, Milomens KMP, Diógenes MAR. Déficits de autocuidado em clientes com diabetes mellitus gestacional: uma contribuição para a enfermagem. Revista Gaúcha de Enfermagem. 29(3):374-381, 2008. Disponível em: https://seer.ufrgs.br/ RevistaGauchadeEnfermagem/article/view/6757

27. Reis CK, Guiraldello EB, Campos CJG. O indivíduo renal crônico e as demandas de atenção. Revista Brasileira de Enfermagem. 61(3):336-341, 2008. Disponível em: http:// www.scielo.br/scielo.php?pid=S0034-71672008000300010\&script=sci_abstract $\&$ tlng =pt 